\title{
Excitability modulation of the motor system induced by transcranial direct current stimulation: A multimodal approach
}

\author{
Maria Concetta Pellicciari a, Debora Brignani a , Carlo Miniussi a,b,* \\ a Cognitive Neuroscience Section, IRCCS Centro San Giovanni di Dio Fatebenefratelli, Brescia, Italy \\ ${ }^{\mathrm{b}}$ Department of Clinical and Experimental Sciences, National Institute of Neuroscience, University of Brescia, Brescia, Italy
}

\section{A R T I C L E I N F O}

Article history:

Accepted 29 June 2013

Available online 9 July 2013

\section{Keywords:}

tDCS

Motor cortex

Cortical reactivity

TMS

EEG

Reaction time

\begin{abstract}
A B S T R A C T
Anodal and cathodal transcranial direct current stimulations (tDCS) are both established techniques to induce cortical excitability changes. Typically, in the human motor system, such cortical modulations are inferred through changes in the amplitude of the motor evoked potentials (MEPs). However, it is now possible to directly evaluate tDCS-induced changes at the cortical level by recording the transcranial magnetic stimulation evoked potentials (TEPs) using electroencephalography (EEG).

The present study investigated the modulation induced by the tDCS on the motor system. The study evaluates changes in the MEPs, in the amplitude and distribution of the TEPs, in resting state oscillatory brain activity and in behavioral performance in a simple manual response task. Both the short- and long-term tDCS effects were investigated by evaluating their time course at $\sim 0$ and 30 min after tDCS.

Anodal tDCS over the left primary motor cortex (M1) induced an enhancement of corticospinal excitability, whereas cathodal stimulation produced a reduction. These changes in excitability were indexed by changes in MEP amplitude. More interestingly, tDCS modulated the cortical reactivity, which is the neuronal activity evoked by TMS, in a polarity-dependent and site-specific manner. Cortical reactivity increased after anodal stimulation over the left M1, whereas it decreased with cathodal stimulation. These effects were partially present also at long term evaluation.

No polarity-specific effect was found either on behavioral measures or on oscillatory brain activity. The latter showed a general increase in the power density of low frequency oscillations (theta and alpha) at both stimulation polarities.

Our results suggest that tDCS is able to modulate motor cortical reactivity in a polarity-specific manner, inducing a complex pattern of direct and indirect cortical activations or inhibitions of the motor system-related network, which might be related to changes in synaptic efficacy of the motor cortex.
\end{abstract}

(c) 2013 Elsevier Inc. All rights reserved.

\section{Introduction}

Several studies have endorsed the use of transcranial direct current stimulation (tDCS), a non-invasive brain stimulation technique, to modulate cortical excitability (Nitsche et al., 2008) and induce neuroplasticity that is associated with cognitive and behavioral changes (Arul-Anandam and Loo, 2009; Boggio et al., 2007; Miniussi et al., 2008; Wagner et al., 2007; Wassermann and Grafman, 2005). As directly shown in animal studies, anodal tDCS increases cortical excitability, inducing a depolarization of the resting membrane potential and increasing neuronal firing rates. In contrast, cathodal tDCS decreases cortical excitability, shifting the resting membrane potential

\footnotetext{
Abbreviations: tDCS, transcranial direct current stimulation; TEP, transcranial magnetic stimulation evoked potential; LMFP, local mean field power.

* Corresponding author at: Neuroscience Section, Department of Clinical and Experimental Sciences, University of Brescia, Viale Europa 11, 25123 Brescia, Italy. Fax: + 39 0303717443.

E-mail address: carlo.miniussi@cognitiveneuroscience.it (C. Miniussi).
}

towards hyperpolarization and reducing the firing rate of neurons (Bindman et al., 1964a; Creutzfeldt et al., 1962; Purpura and McMurtry, 1965). The involvement of mechanisms similar to those underlying long-term potentiation (LTP) and long-term depression (LTD) was hypothesized to explain the tDCS induced neuroplasticity after-effects (Liebetanz et al., 2002; Nitsche et al., 2003b; Nitsche et al., 2004). The changes in neurophysiologic excitability induced by tDCS over the human primary motor cortex (M1) and their underlying mechanisms have been indirectly inferred by assessing the modifications in the excitability of the corticospinal tract using transcranial magnetic stimulation (TMS) protocols (Lang et al., 2011; Nitsche and Paulus, 2000; Nitsche and Paulus, 2001; Nitsche et al., 2005; Priori et al., 1998). Specifically, when tDCS is applied over M1, the main effect observed is an increase in the amplitude of the motor evoked potential (MEP) in the contralateral hand muscles after anodal stimulation, and a decrease after the cathodal one (Nitsche and Paulus, 2000; Nitsche and Paulus, 2001). To overcome the limitations of this indirect approach, several studies have focused their attention on other neurophysiologic measurements as surrogate markers of tDCS-induced 
cortical neuromodulatory effects (Brunoni et al., 2011). In this framework, the use of neuroimaging methods, such as functional magnetic resonance (Baudewig et al., 2001; Jang et al., 2009; Kwon et al., 2008; Polania et al., 2012; Stagg et al., 2009a), positron emission tomography (Lang et al., 2005; Paquette et al., 2011), laser doppler flowmetry (Wachter et al., 2011) and electroencephalography (EEG) (Ardolino et al., 2005; Polania et al., 2010a), have provided further evidence of changes in neural activity induced by tDCS. Moreover, it is understood that, by modulating cortical excitability, tDCS can induce both short- and long-term changes in a polarity-specific manner (Nitsche and Paulus, 2001, Nitsche et al., 2003a). Even if these results highlighted further aspects of tDCS' ability to modulate brain activity, no one, to date, has directly demonstrated current polarity-specific changes induced by tDCS on cortical, peripheral and behavioral measures of the primary motor cortex.

Starting from this scenario, we investigated polarity-dependent tDCS-induced effects using a multimodal experimental approach. Motor system changes in excitability were indexed by the following measures: MEPs, TMS-evoked potentials (TEPs), EEG frequency analyses and motor behavioral reaction times (RTs). Data were collected from healthy participants before and after the application of anodal and cathodal stimulations. A further goal of this study was to investigate short and long-lasting tDCS effects by evaluating the time course of induced changes at $\sim 0$ and 30 min after tDCS. We employed TMS-evoked cortical responses (i.e., TEPs) as a novel probe of tDCS-induced cortical excitability changes (Ilmoniemi et al., 1997; Komssi and Kahkonen, 2006; Miniussi and Thut, 2009; Miniussi et al., 2012).

Combining EEG during TMS allowed the assessment of the local impact of tDCS on neural processing through objective measurements of the cortical reactivity, reflecting the direct activation of the cortical neurons at the site of the stimulation. We used this approach to study the reactivity of the motor cortex using the amplitude of the TEPs to test the overall state of the cortex stimulated by tDCS. In addition, we evaluated the tDCS-polarity dependent changes in the stimulated left as well as in the contralateral M1, with the hypothesis that tDCS might, not only induce site-specific, but also, remote (not-site limited) effects.

\section{Materials and methods}

\section{Subjects}

Eighteen healthy participants took part to the study. Two participants were excluded from the analysis due to excessive noise in the EEG recording. The remaining sixteen participants ( 8 males and 8 females) had a mean age of $23.2 \pm 3$ years. None of the participants had a history of neurological, psychological or other relevant medical disease. None of the participants were on CNS-active medication at the time of the experiment and none had any contraindication for TMS (Rossi et al., 2009). The same criteria were also applied for tDCS. In addition, all participants were right-handed according to the Edinburgh Handedness Inventory test (Oldfield, 1971). The study was approved by the CEIOC Ethics Committee of IRCCS Centro San Giovanni di Dio Fatebenefratelli, Brescia, Italy. Informed consent was obtained from all participants before the experiment.

\section{Experimental design}

Each participant took part in two experimental sessions during which they received anodal and cathodal tDCS, respectively. The order of tDCS polarity conditions (anodal vs. cathodal) was counterbalanced among participants. The two experimental sessions were conducted on the same day (morning and afternoon). The schedule was kept constant across participants (11:30 am and 3:30 pm) to control for potential circadian effects (Sale et al., 2007). Fig. 1 shows the experimental protocol.

To re-establish baseline levels of excitability, a 4-hour break between the two tDCS conditions was planned. During the pause between experimental conditions, participants were awake but relaxed, and were allowed to perform their own preferred relaxation activities under direct experimenter supervision.

Both the corticospinal excitability and the cortical reactivity were investigated recording the MEPs and the TEPs respectively, whereas the cortical state, indexed by oscillatory activity, was evaluated recording EEG activity during a resting state. Reaction times (RTs) were recorded during a simple detection task to evaluate the tDCS-induced effects on the behavioral performance.

All the measures were collected for each experimental session before the tDCS (baseline) and at two time points after the tDCS, i.e., immediately after (post 1 ) and 30 min later (post 2).

To provide the baseline measures, each experimental session began with a TEP-MEP block, followed by an EEG block and finally by a behavioral block. In each TEP-MEP block, 100 single TMS pulses were applied at a random inter-stimulus interval of $0.25-0.5 \mathrm{~Hz}$ with an intensity of $110 \%$ of the RMT. The TEP-MEP block lasted approximately 5 min. Each EEG block consisted of 3 min recording during a resting state with eyes open. In the behavioral block, participants performed a simple RT visual detection task that lasted approximately 5 min. After tDCS, TEP-MEP, EEG and behavioral blocks were acquired at post 1 and post 2 .

During the experiment, participants were seated on a dedicated, comfortable armchair in a Faraday-cage, sound-proofed room. During TEP-MEP and EEG blocks, participants were instructed to keep their hands completely relaxed, passively sitting and fixing their eyes on a visual target directly in front of them. Each experimental session lasted approximately $75 \mathrm{~min}$.

\section{$t D C S$}

The stimulation was delivered by a battery-driven electrical stimulator (NeuroConn GmbH, Ilmenau, Germany) through a couple of conductive-rubber electrodes placed inside saline-soaked sponges (electrode surface $25 \mathrm{~cm}^{2}$ ). For anodal and cathodal stimulations, the current was delivered with an intensity of $1 \mathrm{~mA}$ (current density $0.04 \mathrm{~mA} / \mathrm{cm}^{2}$ ) for $13 \mathrm{~min}$, with a ramping period of $8 \mathrm{~s}$ both at the beginning and at the end of the stimulation. The active electrode was placed over the motor cortical representational field of the right first dorsal interosseous muscle (FDI), as defined by means of a TMS mapping experiment (see below). The reference electrode was placed over the right frontopolar cortex (above the eyebrow). The electrodes were oriented approximately parallel to the central sulcus and the eyebrow. This montage was chosen because it has been shown to be effective in modulating corticospinal excitability from M1 in a polarity-specific fashion (Moliadze et al., 2010; Nitsche and Paulus, 2000; Nitsche and Paulus, 2001). The terms "anodal" and "cathodal" refer to the polarity of the electrode placed over the left M1. Before fixing the electrodes with elastic bands, an electro-conductive gel was applied under the saline-soaked sponges to reduce contact impedance. The participants were blind to the tDCS conditions.

\section{TMS}

Single pulse TMS was carried out by a Magstim SuperRapid magnetic stimulator connected to one booster module and a standard figureof-eight shaped coil with an outer winding diameter of $70 \mathrm{~mm}$ (Magstim Company, Whitland, UK) that generates $2.2 \mathrm{~T}$ as a maximum output. In the present protocol, individual biphasic stimuli were employed. The coil was placed tangentially to the scalp, the handle pointing backwards and laterally, about a $45^{\circ}$ angle from the mid-sagittal axis of the participants' heads and oriented to elicit a posterolateral-anteromedial current flow in the brain tissue. The stimulation started at a supra-threshold intensity. The optimal stimulus site to elicit MEPs in the right FDI, termed the "motor hotspot", was identified by positioning the coil approximately over the central sulcus and moving it on the scalp by $0.5 \mathrm{~cm}$ steps on left M1. The hotspot was then marked directly on the scalp 


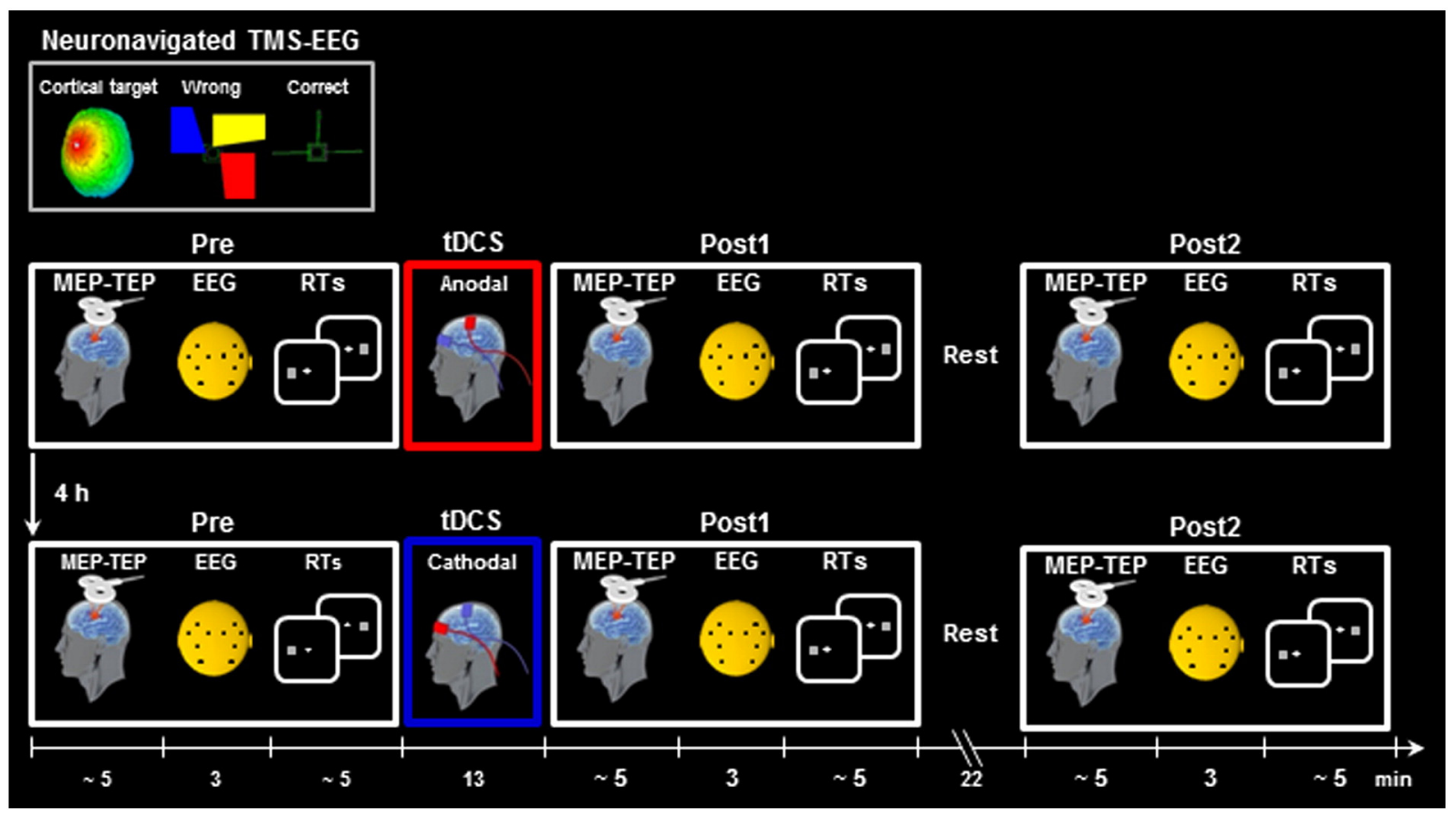

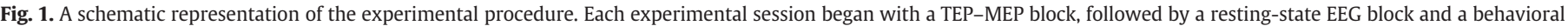

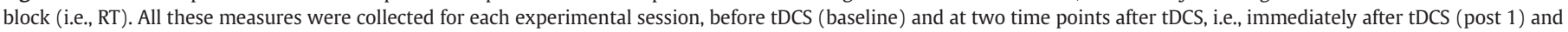

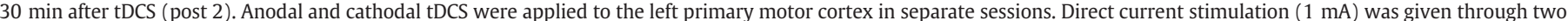

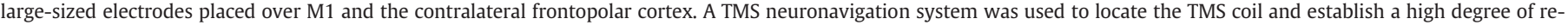
producibility across separate experimental sessions.

with a soft-tip pen. On this site, RMT was assessed as the lowest stimulus intensity needed to produce a response of at least $50 \mu \mathrm{V}$ in amplitude in the relaxed muscle for at least five out of ten consecutive stimulations, at a resolution of $1 \%$ of the maximal stimulator output (Rossini et al., 1994). A TMS neuronavigation system (SofTaxic, E.M.S., Bologna, Italy) was used to ensure a high degree of reproducibility across separate experimental sessions (Carducci and Brusco, 2012; Cincotta et al., 2010).

\section{TEP, EEG and MEP recordings}

A TMS-compatible EEG equipment (BrainAmp 32MRplus, BrainProducts GmbH, Munich, Germany) was used to record the EEG and the electromyography (EMG) (Veniero et al., 2009). To minimize the overheating of the electrodes proximal to the stimulating coil, TMS-compatible $\mathrm{Ag} / \mathrm{AgCl}$ sintered ring electrodes were used. Ten unipolar EEG derivations were recorded from the scalp electrodes. Four electrodes were functionally positioned around the cortical motor hotspot in each orthogonal direction at $3 \mathrm{~cm}$ distance (anteriorly, posteriorly, laterally to the left and laterally to the right). Four additional electrodes were positioned over the right hemisphere, mirroring the contralateral set-up. The last two electrodes were placed over P3 and P4, according to the 10-20 International System. Additional electrodes were used as the ground and reference. The ground electrode was placed in mid-occipital $(\mathrm{Oz})$ position. The right mastoid served as reference for all electrodes. Recordings obtained from the left mastoid electrode were used off line to re-reference the scalp recordings to the average of the left and the right mastoid, i.e., including the implicit reference (right mastoid) into the calculation of the new reference. Horizontal and vertical eye movements were detected by recording the electrooculogram (EOG), to monitor participant behavior on line and to reject off-line the trials with ocular artifacts. Surface EMG activity was recorded from the right FDI muscle, with the active electrode mounted on the belly of the muscle and the reference electrode placed over the base of the metacarpo-phalangeal joint. The EMG activity was monitored throughout the experiment to guarantee a complete muscle relaxation.

The EEG, EOG and EMG signals were acquired with a band-pass filter at $0.1-1000 \mathrm{~Hz}$ and digitized at a sampling rate of $5 \mathrm{kHz}$ using a 16 bit A/D-converter. Skin/electrode impedance was kept below $5 \mathrm{k} \Omega$. Data were analyzed offline with dedicated software (Brain Vision Analyzer, Brain Products GmbH, Munich, Germany).

To analyze cortical and peripheral responses to TMS, the continuous EEG and EMG signals were divided off line into epochs (from $100 \mathrm{~ms}$ before to $300 \mathrm{~ms}$ after referred to each TMS pulse) and were baseline corrected (i.e., we normalized the baseline activity at each site to a $0-\mu \mathrm{V}$ level from $100 \mathrm{~ms}$ pre-stimulus to $0 \mathrm{~ms}$ ). Before averaging, epochs containing eye blinks (signal above $100 \mathrm{mV}$ ) or muscle artifacts were rejected. The TMS-induced artifact was not removed from the recording (see Veniero et al., 2009). It is important to note that if an EEG epoch was rejected, the corresponding EMG epoch was also rejected (Ilmoniemi and Kicic, 2010), resulting in a one-to-one correspondence between cortical and peripheral measures.

To analyze the TEPs, the EEG sampling rate was reduced to $1000 \mathrm{~Hz}$. The cortical evoked responses to TMS (i.e., TEPs) were averaged before and after each tDCS condition, separately for each time point (baseline, post 1 and post 2 ) in the whole epoch (from -100 before to $+300 \mathrm{~ms}$ after the TMS pulse) and for each participant. To evaluate tDCS-induced local excitability changes, two regions of interest (ROI) were selected in each hemisphere: on the motor cortical hotspot (four electrodes around the stimulated left M1) and on the contralateral homologue area (the corresponding electrodes from the right hemisphere).

To determine the tDCS-induced changes in the cortical field strength evoked by the TMS, a local mean field power (LMFP) analysis was computed. LMFP represents a measure of local brain activation strength at a given time point in a given scalp area (Casarotto et al., 2013). This local index was calculated as the root-mean-square value of the signal across the ROI electrodes (motor cortical hotspot), separately for each hemisphere. The LMFP values were computed for each current polarity condition and for each time point in the whole epoch. 
To characterize the tDCS-induced changes in the cortical oscillatory activity, the EEG data acquired during the resting state - before and after each tDCS condition - were analyzed off line, separately for each time point (baseline, post 1 and post 2). Continuous EEG recordings were segmented in 2-second epochs and those with excessive drift, eye movements, blinks or muscle artifacts were excluded from the analysis. Power density was estimated by means of the fast Fourier transform (10\% Hanning-window; frequency resolution $1 \mathrm{~Hz}$ ) for all the frequencies ranging from 2 to $45 \mathrm{~Hz}$, and divided into five bands as follows: $2-4 \mathrm{~Hz}$ (delta), $5-7 \mathrm{~Hz}$ (theta), $8-12 \mathrm{~Hz}$ (alpha), $13-30 \mathrm{~Hz}$ (beta) and $31-45 \mathrm{~Hz}$ (gamma). The mean band power was then obtained by averaging the power values of all the single-trial epochs for each participant.

\section{Behavioral task}

To investigate if the tDCS affects the speed of the motor response, RTs were collected during a simple detection task. During the task, participants were sitting in front of a computer screen placed at eye level and were asked to fix a small cross at the center of the screen and to press a centrally located key with their index finger as rapidly as possible following stimulus onset. The stimuli consisted of light gray squares $\left(1.4^{\circ}\right)$ presented on a black background for $50 \mathrm{~ms}$. The stimuli appeared, in a random sequence, on the right or on the left with respect to the central fixation cross. Each participant performed 2 blocks and the response hand alternated after each block, with the order counter-balanced between participants. Each block consisted of 20 trials for each hemifield, for a total of 40 trials. The aim was to verify whether the tDCS effects were specific for the hand contralateral to the side of stimulation (i.e., the right hand) or whether there was a generalization to both hands.

\section{Sensations induced by tDCS}

At the end of each tDCS stimulation, the participants completed a questionnaire about sensations experienced during the two stimulations (anodal vs. cathodal) to evaluate whether the tDCS caused any discomfort (Fertonani et al., 2010).

\section{Data analysis and results}

\section{Sensations}

During tDCS sessions, mild itching and/or irritation were the most commonly reported sensations (in $72 \%$ and in $66 \%$ of participants, respectively). Notably, there were no differences between the sensations experienced during the anodal and the cathodal tDCS, as shown by the nonparametric Wilcoxon rank sum tests (all ps $>0.18$ ).

\section{Corticospinal excitability - MEP}

The changes in the corticospinal excitability induced by the tDCS were evaluated using the MEP amplitudes as the dependent variable. The amplitude of each MEP was measured peak-to-peak, and the mean values were calculated before (baseline) and after (post 1 and post 2) each tDCS polarity condition. Two separate repeated-measures ANOVAs were performed. The first ANOVA on short term effects, with factor tDCS polarity (anodal vs. cathodal) and time (baseline vs. post 1 ), and the second on long term effects with factor tDCS polarity (anodal vs. cathodal) and time (baseline vs. post 2). Bonferroni post-hoc corrections were performed for significant comparisons.

\section{Short-term effects}

The analysis performed at time 0 after the tDCS showed a significant interaction between the tDCS polarity and time $\left(\mathrm{F}_{1,15}=27.5\right.$; $\mathrm{p}<0.001$ ), revealing that the MEPs were significantly modulated by the tDCS polarity and confirming the effectiveness of the experimental manipulation, as shown in Fig. 2a. In line with previous studies, the application of the anodal tDCS over M1 induced a short-term enhancement of corticospinal excitability, whereas cathodal stimulation produced a short-term reduction, as indexed by increased and decreased MEP amplitudes respectively. The post-hoc analyses showed that the MEP amplitudes increased $+30.69 \pm 9.6 \%$ following the anodal $(\mathrm{p}=0.01)$ stimulation and decreased $-24.2 \pm 5.5 \%$ following the cathodal stimulation $(p=0.01)$, compared with the baseline (see Fig. 2b). Moreover, after tDCS, we found a clear and significant difference in the mean MEP amplitudes between the two tDCS conditions (after anodal $884.25 \mu \mathrm{V}$ and after cathodal $535.32 \mu \mathrm{V}$; $\mathrm{p}<0.001)$.

\section{Long-term effects}

The analysis of the tDCS-induced, long-term effects in cortical excitability (measured $30 \mathrm{~min}$ after the stimulation) showed a marginally significant interaction between the tDCS polarity and time $\left(\mathrm{F}_{1,15}=4.25 ; \mathrm{p}=0.056\right)$. The changes in the MEPs persisted after the anodal stimulation, with an increase of $+34.35 \pm 8.66 \%$ $(\mathrm{p}<0.01)$, but not after the cathodal stimulation $+9.65 \pm 12.99 \%$ $(\mathrm{p}=1)$.

\section{Cortical reactivity - TEP}

To investigate whether the tDCS induces any short-term and long-term polarity-dependent changes in the cortical responses, we performed a Student's t test (paired samples, two tailed) first, comparing the mean values acquired during the baseline with those acquired during post 1 or post 2. These analyses were performed separately for each tDCS polarity (anodal vs cathodal) and for each hemisphere (right vs. left, respectively contralateral and ipsilateral to the stimulated hotspot) for each time point following the TMS pulse. In short, we wanted to determine the specific effects and time course of the LMFP responses acquired at baseline compared with those acquired after the stimulation (at post 1 and post 2).

The anodal and cathodal stimulations applied over M1 induced definite modulations of the cortical reactivity, at precise time windows. The analyses revealed an immediate increase in excitability after the anodal stimulation over both hemispheres. The cathodal stimulation induced opposite effects over the two hemispheres, with reduced excitability over the stimulated hemisphere and facilitation in the contralateral one. The same cortical reactivity changes observed immediately after tDCS application were observed at post 2 .

\section{Short-term effects}

After the anodal stimulation, a significant increase in the cortical reactivity was observed at specific time intervals over both the stimulated left (intervals 20-27 ms, 51-72 ms, 258-265 ms; all ps $<0.05$ ) and right (intervals 10-16 ms, 86-96 ms, 209-231 ms; all ps < 0.05) hemispheres. These results highlight the inter-hemispheric spread of cortical activation induced by anodal stimulation (see Figs. $2 \mathrm{~d}$ and 3 ).

After the cathodal stimulation, two different effects were observed between the hemispheres: a late reduction in cortical reactivity over the stimulated left hemisphere (interval 206-238 ms; all ps $<0.05$ ) and an increase in cortical reactivity over the right hemisphere at the early and middle time windows (intervals 37-41 ms, 124-152 ms; all ps $<0.05$ ) (see Figs. 2e and 3).

\section{Long-term effects}

The anodal stimulation induced diffuse increases in the cortical reactivity over both the left $(15-33 \mathrm{~ms}, 54-75 \mathrm{~ms}$; all ps $<0.05)$ and the right (86-96 ms, 205-233 ms; all ps $<0.05$ ) hemispheres. The cathodal stimulation induced a significant long-lasting cortical reactivity decrease over the left hemisphere $(201-217 \mathrm{~ms}$; all ps $<0.05)$ 
a

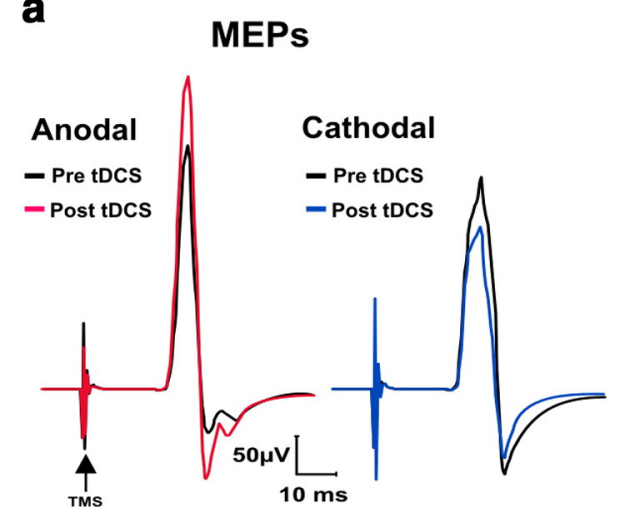

e b

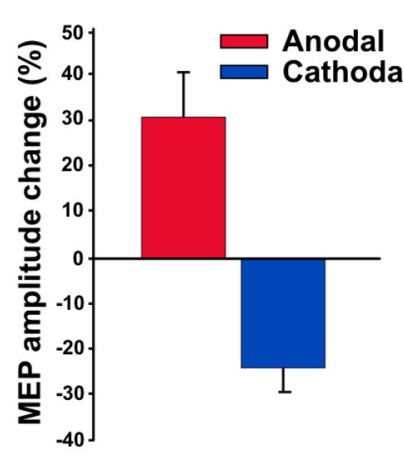

Anodal

- Pre tDCs

- Post tDCS
C

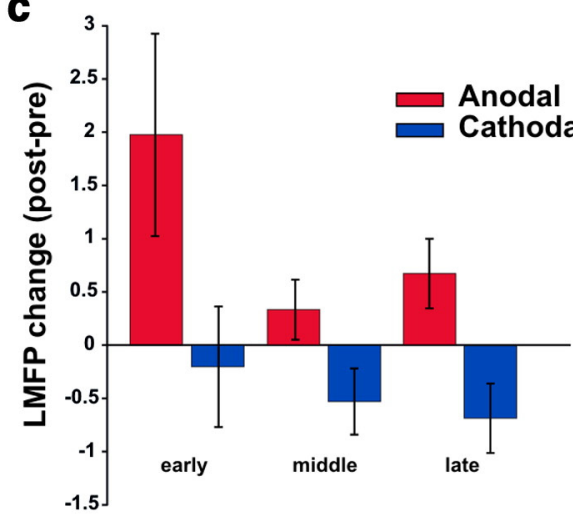

TEPs d
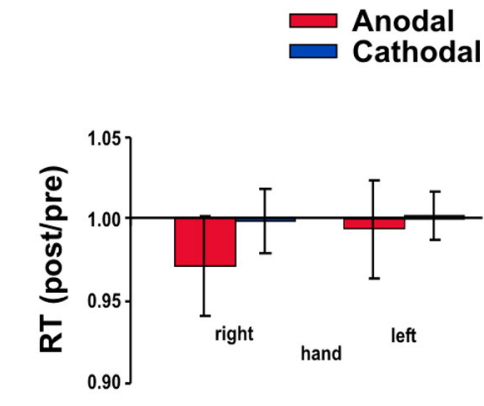

Cathodal

- Pre tDcs

- Post tDCS
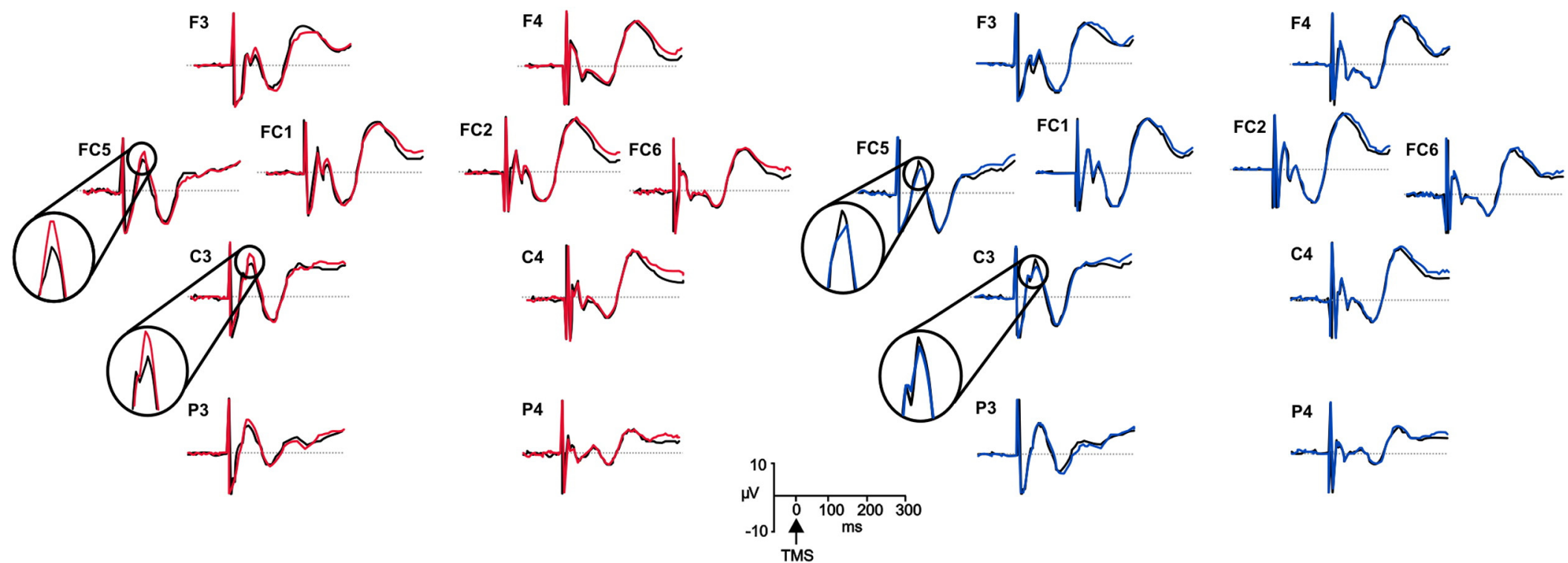

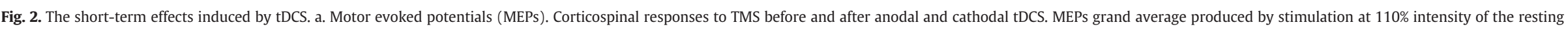

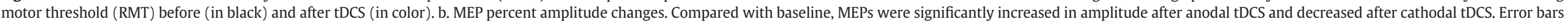

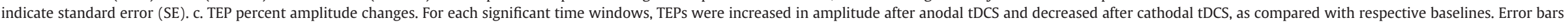

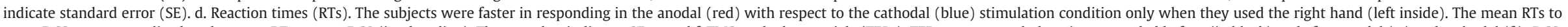

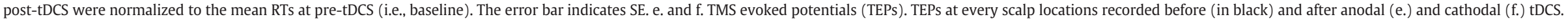



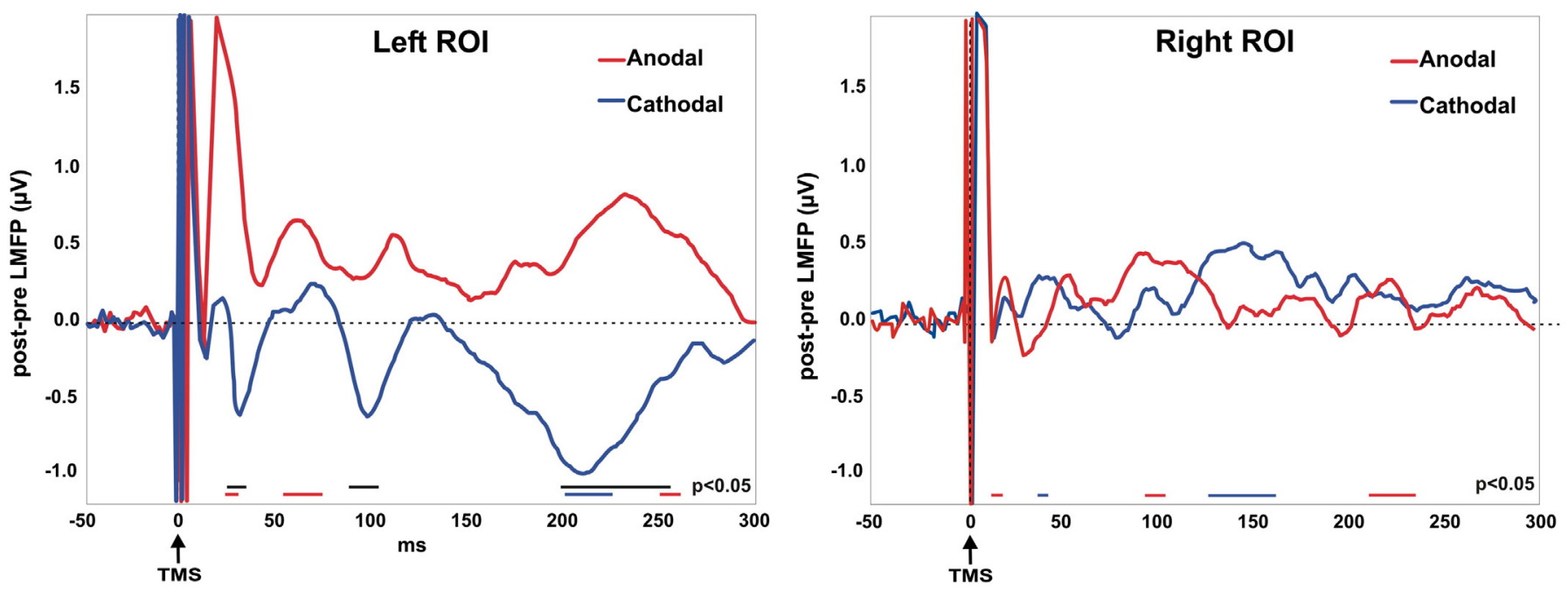

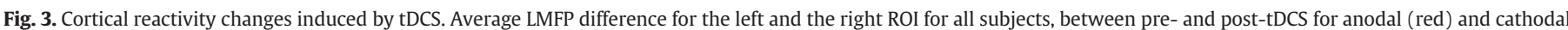

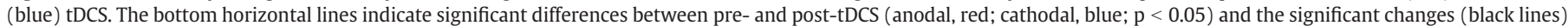
in the direct comparison of the difference between pre- and post-tDCS for the two conditions. The black arrows indicate TMS pulse.

and a cortical reactivity increase over the right hemisphere (3439 ms, 124-131 ms; all ps < 0.05).

These results suggest that the cortical reactivity changes induced by the anodal and cathodal tDCS lasted for a long time, involving not only the site of stimulation but also the contralateral side.

In a second analysis, we directly compared the changes induced by the anodal and cathodal stimulations. Two separate repeatedmeasures ANOVAs were conducted at each time point in the whole epoch after the TMS pulse, one for post 1 and one for post 2 . The LMFP differences between the baseline and those following the tDCS were used as dependent measures, testing for the tDCS polarity (anodal vs. cathodal) and the hotspot (stimulated hemisphere vs. contralateral one). Post-hoc paired $t$-tests were performed on significant comparisons. The analyses revealed a significant difference between the anodal and cathodal stimulations only in the left hemisphere, at different time intervals. In particular, the anodal stimulation induced an increase and the cathodal stimulation induced a decrease in the cortical reactivity over the stimulated hemisphere. Overall, we observed these results in the short term and partially in the long term.

\section{Short-term effects}

Our analyses highlighted the specific time-course of the short-term cortical reactivity changes induced by the tDCS polarities, revealing significant effects in three specific time windows: early (from 22 to $32 \mathrm{~ms}$ ), middle (from 92 to $105 \mathrm{~ms}$ ) and late (from 200 to $264 \mathrm{~ms}$ ) (see Fig. 3).

A significant effect of the tDCS polarity was observed in all three time windows (all $\mathrm{F}_{1,15}>4.67$, p values $<0.05$ ). Notably, a robust interaction between the tDCS polarity and the hemisphere was found only in the early and late windows (all $\mathrm{F}_{1,15}>4.61$, p values $<0.05$ ), showing a specific pattern of current-dependent changes on the stimulated area. No significant effect of the hemisphere was observed. Post-hoc analyses revealed consistent differences between the anodal and cathodal stimulations on the stimulated hemisphere in all time windows (all $\mathrm{t}_{15}>2.138$, $\mathrm{p}$ values $<0.05$ ) and no differences in the contralateral hemisphere, highlighting that the specific tDCS polarity effects on the cortical reactivity are limited to the stimulated area. Moreover, the anodal stimulation induced an early increase (all $\mathrm{t}_{15}>2.137$, $\mathrm{p}$ values $<0.05$ ), whereas the cathodal stimulation generated a middle and late decrease (all $t_{15}>2.132$, $\mathrm{p}$ values $<0.05$ ) in the cortical reactivity over the left hemisphere when compared with the right one.

\section{Long-term effects}

The analysis of the cortical reactivity changes induced by the tDCS highlighted long-lasting effects in all three time windows, partially overlapping those observed in the short-term analyses (from 16 to $40 \mathrm{~ms}$; from 59 to $81 \mathrm{~ms}$; from 182 to $235 \mathrm{~ms}$ ).

A main effect of the tDCS polarity was observed only in the late time window (all $\mathrm{F}_{1,15}>4.56$, p values $<0.05$ ), whereas a significant interaction between the tDCS polarity and the hemisphere was found in both the early and the late time windows ( $a l l \mathrm{~F}_{1,15}>4.54$, $\mathrm{p}$ values $<0.05$ ), showing a stable time pattern of current-specific changes on the stimulated area. In addition, a main effect of the hemisphere was significant in all time windows (all $\mathrm{F}_{1,15}>4.68$, p values $<0.05$ ).

The post-hoc analyses revealed that the tDCS induced long-lasting, polarity-specific differences over the left (stimulated) hemisphere (all $t_{15}>2.157$, $p$ values $<0.05$ ), in the early and late time intervals, but not over the contralateral hemisphere. Moreover, the anodal stimulation induced an early and middle increase in the cortical reactivity (all $\mathrm{t}_{15}>2.194$, $\mathrm{p}$ values $<0.05$ ), whereas the cathodal stimulation induced an early and late decrease (all $t_{15}>2.143$, p values $<0.05$ ) over the stimulated left hemisphere if compared to the right one.

\section{EEG activity during resting state}

For statistical analysis, power density measures were logtransformed to obtain a Gaussian distribution of data suitable for repeated measures tests. Two separate repeated-measures ANOVAs were performed. An ANOVA was applied to evaluate the short term effects induced by the tDCS on the ongoing brain activity, testing at each frequency band the following factors: tDCS polarity (anodal vs. cathodal), time (baseline vs. post1), hemisphere (right vs. left, respectively contralateral and ipsilateral to the stimulated hotspot) and electrode site (5 levels). Subsequently, a second ANOVA was applied on the factors: tDCS polarity (anodal vs. cathodal), time (baseline vs. post 2), hemisphere (right vs. left, respectively contralateral and ipsilateral to the stimulated hotspot) and electrode site (5 levels), to test the tDCS induced-long term effects. The Greenhouse-Geisser epsilon correction factor was applied, where appropriate, to compensate for possible effects of non-sphericity in the measurements. Bonferroni post-hoc correction was performed on significant comparisons (Fig. 4).

\section{Short-term effects}

The comparison between the power densities acquired at the baseline and at post 1 revealed no specific effects related to the tDCS 


\section{Anodal}

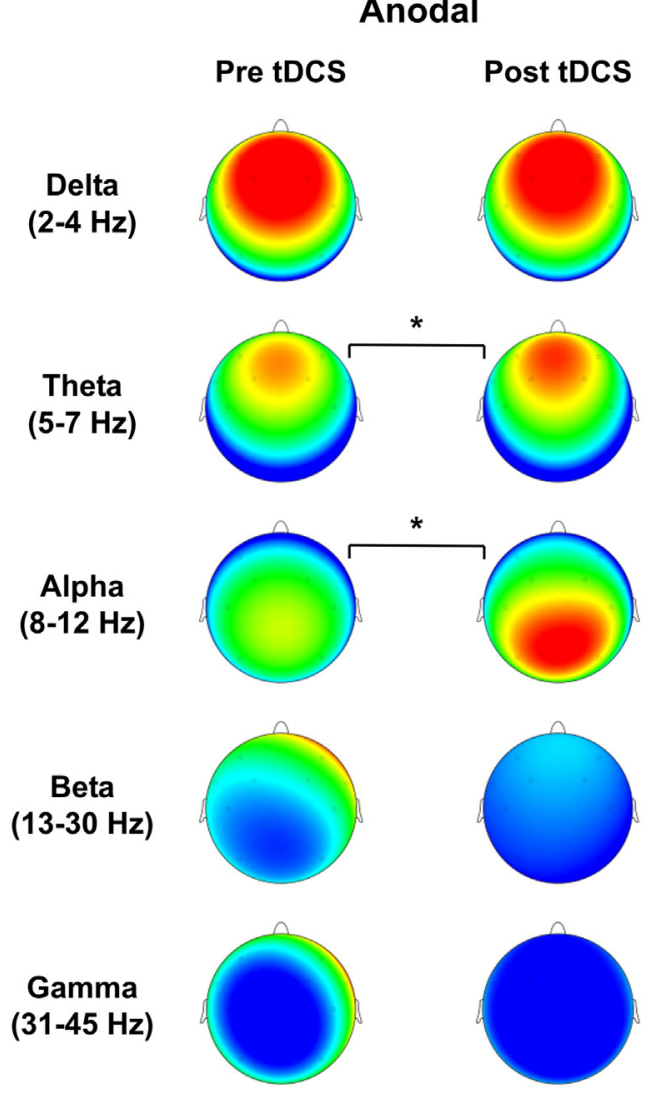

Cathodal

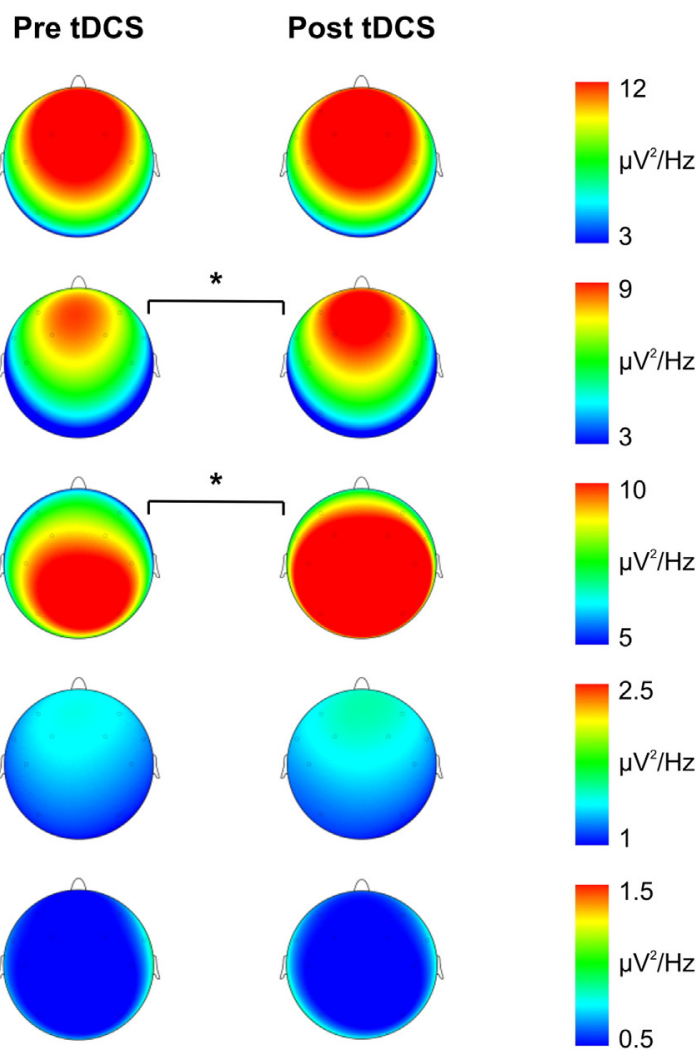

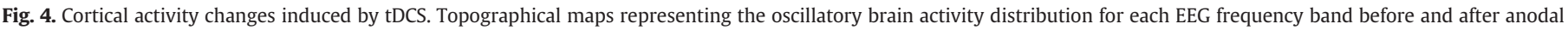

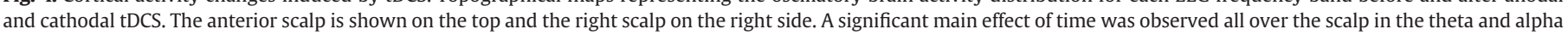
bands, with no polarity-specific effects.

polarity (all $\mathrm{F}<2.97$, $\mathrm{p}$ values $>0.1$ ). There was a significant main effect of time in the theta $\left(F_{1,15}=5.26 ; p=0.037\right)$ and alpha $\left(F_{1,15}=10.82\right.$; $\mathrm{p}=0.005$ ) bands, but this effect showed no interaction with other factors (all $\mathrm{F}<2.97$, $\mathrm{p}$ values $>0.1$ ), suggesting an equivalent increase of power density after both the anodal and the cathodal stimulations over all the scalp sites. No significant main effect or time interactions were found in the delta, beta or gamma bands (all $F<2.59$, $\mathrm{p}$ values $>0.08$ ).

\section{Long-term effects}

The tDCS-induced changes in the cortical oscillatory activity were reduced 30 min after the stimulation. A general increase in the power density was still observable after the anodal stimulation only in the alpha band, as revealed by the interaction between time and the tDCS polarity $\left(F_{1,15}=5.26 ; p=0.037\right)$. A new time-related effect emerged in the delta band, that is, the power density decreased after the cathodal stimulation over the right hemisphere only [time $x$ tDCS polarity $\times$ hemisphere $\left(\mathrm{F}_{1,15}=5.16 ; \mathrm{p}=0.038\right)$; time $\times$ tDCS polarity $\times$ hemisphere $\times$ electrode site $\left(\mathrm{F}_{4,60}=4.57 ; \mathrm{p}=0.02\right.$ ]. No significant effects were found in the theta, beta or gamma bands (all $\mathrm{F}<1.48$, $\mathrm{p}$ values $>0.2$ ).

Behavioral task $-R T$

Anticipations (i.e., pressing the spacebar before the appearance of the target), omissions (no response) and RTs shorter or longer than \pm 2 standard deviations were excluded from the analyses. The error rate ( $2.6 \%$ of the trials) was submitted to a statistical analysis in order to identify any response difference between the tDCS polarities. A paired t-test comparing post 1 errors normalized to the baseline revealed no differences between the anodal and the cathodal stimulations $\left(t_{15}=1.45\right.$, $\mathrm{p}=0.17)$. Two separate repeated-measures ANOVAs were conducted on the mean RTs with tDCS polarity (anodal vs. cathodal), hand (left vs. right) and time (baseline vs. post tDCS) as within-subject factors. To assess any significant interactions, we performed selected twosample comparisons by means of the $t$-tests, using the Bonferroni corrections where appropriate.

\section{Short-term effects}

The statistical analysis revealed a significant interaction between the tDCS polarity and the hand $\left(\mathrm{F}_{1,15}=5.67, \mathrm{p}=0.03\right)$, showing a specific effect for the hand used in the task. Participants responded more rapidly during the anodal session only when they used the right hand, as shown in Fig. 2c. The right hand was contralateral to the side of the stimulation. No differences were observed when participants used their left hand. The lack of significant interactions between the tDCS polarity and hand with the time factor suggests, however, that this result may not be ascribed to the stimulation in itself.

To explore the relationship between the right-hand responses and the corticospinal excitability, we performed a simple linear regression analysis between the right-hand RTs and the MEP amplitudes, correcting for the differences between the anodal and the cathodal tDCS at post 1 . A significant negative correlation was found $\left(\mathrm{r}=-0.55, \mathrm{t}_{14}=-2.44, \mathrm{p}=0.03\right)$, suggesting that the RTs were inversely related to the MEP amplitude.

\section{Long-term effects}

Contralateral hand specificity disappeared 30 min after the end of the stimulation. No significant differences persisted between the right and the left hand (all $\mathrm{F}<3.28$, $\mathrm{p}$ values $>0.09$ ). A trend, however, was observed for the time $\left(\mathrm{F}_{1,15}=4.36 ; \mathrm{p}=0.054\right)$, suggesting that subjects were faster in responding at post 2 compared to the baseline, 
regardless of hand or tDCS polarity. Therefore, this marginal improvement in response times likely reflected a general learning effect that was not tDCS-related. Indeed, it is very likely that the task itself could be learned, producing an inevitable improvement in the performance by the end of the experiment.

Correlation between corticospinal excitability and cortical reactivity changes

To evaluate whether the corticospinal excitability could be related to the cortical responses evoked by the TMS, the individual MEP changes induced by the tDCS and the individual LMFP changes over the stimulated left motor cortex were subjected to a correlation analysis.

To reduce the total number of possible correlations, only the individual LMFP changes of the significant time windows, separately for anodal and cathodal tDCS sessions were considered. Specifically, the pre-post MEP percent changes were correlated with the pre-post LMFP percent changes of the following time windows: 20-27 ms (early window), 51-72 ms (middle window) and 258-265 ms (late window), for the anodal condition; and 206-238 ms for cathodal condition. No reliable correlation was found between the mean amplitude of the MEPs and the TMS-induced cortical responses both after anodal (Pearson's correlation coefficients: $r=0.08$ for MEPs/early window; $r=0.26$ for MEPs/middle window; $r=0.29$ for MEPs/late window) or the cathodal stimulation ( $\mathrm{r}=-0.32$ for MEPs/late window).

Further, in line with a previous study (Huber et al., 2008) and to determine to what extent the MEP changes predicted the LMFP changes in the direct comparison of the difference between preand post-tDCS across both conditions, the MEP amplitude change was correlated with the change of the LMFP integrated activity, recorded over the left M1. No significant correlation was found between the MEP and the LMFP changes $(r=-0.15)$. Our results are in agreement with previous studies that, comparing cortical and corticospinal responses before and after several plasticity induction protocols (Van der Werf and Paus, 2006; Esser et al., 2006), shows no correlation between these measures. The weak correlations between the MEPs and the TMS evoked cortical potentials (Huber et al., 2008) or the total corticospinal volley (Lang et al., 2011) after the plasticity induction protocols, suggest that MEPs should not be considered as an unambiguous marker of the cortical excitability changes.

As such, we hypothesize that the lack of strong correlations between the MEP and the LMFP changes after the tDCS could be due to the high variability in MEPs' amplitude. Such variability is given, not only by cortical excitability variability, but also by a specific contribution of the spinal motoneuronal pools to the MEP amplitude not involved in determining the cortical evoked response (Bonato et al., 2006). One other factor that should be considered relates to the difference in the latency between the MEP and the cortical reactivity effects. The physiological MEP latency is $\sim 20 \mathrm{~ms}$ whereas our cortical changes were present later in latency.

We conclude that, in addition to the MEPs, the TEPs represent a further surrogate marker of tDCS-induced neuromodulations, measuring specifically the cortical activation and its changes. Future correlation studies between the MEP and the TEP component changes at the single trial level (Mäki and Ilmoniemi, 2010) or between the MEPs and the weighted TEP (Vernet et al., 2013) are necessary to understand the relationship between the tDCS-induced effects on the corticospinal and the cortical excitability and their measures.

\section{Carry over effects of stimulation order}

In light of recently published studies (Fricke et al., 2011; Monte-Silva et al., 2010; Monte-Silva et al., 2013), the impact of the tDCS on the neuromodulation mechanisms may differ depending on the length of the intervals between the stimulations. Monte-Silva et al. (2013) showed that the application of a second period of stimulation, of the same polarity, during the after-effects of a first period, causes a modification in the amplitude of MEPs.

Therefore, to verify the presence of long lasting effects on the present protocol, we performed further analyses, taking into consideration the stimulation order on the RMT, the MEP amplitude and the EEG activity at the baseline level as well as after the tDCS. All the results reported are expressed as mean and standard error (SE).

As a first step, to evaluate if the cortical and corticospinal excitability differed at the time of the two baseline measures (before the anodal and before the cathodal stimulations) and if any excitability changes induced by the first tDCS session affected the second one, repeated measures ANOVAs were applied respectively to the RMT measured at the beginning of each experimental session, to the baseline MEP amplitudes and to the EEG activity, testing the tDCS condition (anodal vs. cathodal) as a within-subject factor and the order of stimulation (anodal-cathodal vs. cathodal-anodal) as a between-subject factor. The statistical analyses highlighted the stability of corticospinal excitability before both the tDCS conditions. There were no significant differences in the RMT acquired before the anodal or cathodal conditions, as revealed by non-significant effects of the stimulation order $\left(\mathrm{F}_{1,14}=0.42\right.$; $\mathrm{p}=0.52)$ and the tDCS condition $\left(\mathrm{F}_{1,14}=2.72 ; \mathrm{p}=0.12\right)$ and no order by the tDCS condition interaction $\left(\mathrm{F}_{1,14}=0.009 ; \mathrm{p}=0.92\right)$. The RMT was $50.6 \pm 1.48 \%$ of the maximum stimulator output before the anodal and $51.6 \pm 1.86 \%$ before the cathodal tDCS.

Similarly, the analyses of the MEP amplitude acquired before anodal or cathodal conditions highlighted no significant differences, as reflected in the non-significant effects of the stimulation order $\left(F_{1,14}=0.089\right.$; $\mathrm{p}=0.76)$, the tDCS condition $\left(\mathrm{F}_{1,14}=0.28 ; \mathrm{p}=0.60\right)$ or order by the tDCS condition interaction $\left(\mathrm{F}_{1,14}=1.30 ; \mathrm{p}=0.27\right)$.

Finally, the ANOVA on the EEG frequencies revealed no significant effect due to the main factor order (all $\mathrm{F}<1.21$; $\mathrm{p}$ values $>0.29$ ) or to the interactions between the order and other factors $(\mathrm{F}<3.47$; $\mathrm{p}$ values $>0.06$ ), suggesting no difference between participants who received anodal the tDCS or those who received the cathodal tDCS as first.

Subsequently, in order to exclude the influence of the order effect on the mean analysis results, we performed additional analyses on the MEP amplitude and the EEG power densities after the tDCS, testing the tDCS condition (anodal vs. cathodal) as a within-subject factor and the order effect (anodal-cathodal vs. cathodal-anodal) as a between-subject factor.

For the MEP amplitude after the tDCS, the results of the ANOVA highlighted no significant order effect $\left(\mathrm{F}_{1,14}=0.12 ; \mathrm{p}=0.73\right)$ and no order by the tDCS condition interaction $\left(\mathrm{F}_{1,14}=0.13 ; \mathrm{p}=0.72\right)$. We found only a significant effect of the tDCS condition $\left(\mathrm{F}_{1,14}=10.28\right.$; $\mathrm{p}=0.0063$ ) that revealed a tDCS polarity-dependent effects on the MEP amplitude, in line with the main hypotheses.

The ANOVA on percent changes of the MEP amplitude (before and after tDCS) highlighted no significant effects of order $\left(F_{1,14}=0.034\right.$; $\mathrm{p}=0.85)$ and no order by the tDCS condition interaction $\left(\mathrm{F}_{1,14}=\right.$ $2.53 ; \mathrm{p}=0.13)$. We found only a significant effect of the tDCS condition $\left(F_{1,14}=43.06 ; p=0.000013\right)$ that revealed a difference in the MEP amplitude changes induced by the tDCS condition, as predicted by the main hypotheses.

For the EEG data, we tested the influence of the order of the stimulation (anodal-cathodal vs. cathodal-anodal) on the power densities acquired after the tDCS, considering factors such as: tDCS polarity (anodal vs. cathodal), hemisphere (right vs. left) and electrode site (5 levels). The analyses revealed no significant effect due to the order or to interactions between order and other factors ( $\mathrm{F}<1.45$; $\mathrm{p}$ values $>0.2$ ).

These results led us to assume that the used stimulation parameters did not induce corticospinal and cortical excitability changes lasting longer than $4 \mathrm{~h}$ supporting the use of two tDCS polarities on the same day over the same subject. 


\section{Limitations}

With regard to the TEP session, no white noise was used to mask the coil-generated click, to avoid discomfort to the subjects. This noise must be of high intensity to partially cover the coil-generated click, considering that the bone-conducted coil-generated click cannot be canceled. Moreover, no sham stimulation was applied because we assumed that the neurophysiological and behavioral changes observed in our study were not due to a placebo effect. Our participants were blind to the current polarity stimulation condition and its expected excitability shifts.

It should be hypothesized that EEG alterations without an additional control condition (sham) might also be due to stimulation-independent effects like fatigue and practice instead of the stimulation polarity. Nevertheless, this aspect can partially be ruled out because the stimulation order was balanced among the subjects. Finally, the limited number of recording electrodes prevented the detection of temporalspatial dynamics of the cortical spread of activation/deactivation induced by different current polarity stimulations, limiting the information on the topographical effects induced by the tDCS. For the same reason, we could not establish if the modulation in the recording area was due to changes in the neighboring cortical areas, indirectly modified by the tDCS spreading to the recording area by volume conduction.

\section{Discussion}

In this study, we demonstrated that the tDCS applied over M1 is able to induce, in a polarity-specific manner, neuromodulatory effects on the corticospinal and cortical excitability indices, but not on the oscillatory brain activity and on the motor behavioral performance.

Specifically, the application of the anodal tDCS induced an enhancement of the corticospinal excitability, whereas the cathodal stimulation produced a reduction, as indexed by changes in the MEP amplitudes. These results were also corroborated by the TEP analyses. The anodal stimulation induced an increase in the motor cortical reactivity while the cathodal stimulation induced a decrease. These site-specific changes on the cortical reactivity were clearly present at short-term and after $30 \mathrm{~min}$, although in a reduced manner. Moreover, when we directly compared the cortical activation induced by the TMS, separately in the left and right hemispheres, we found that both the anodal and the cathodal tDCS induced an overall excitability increase compared to the baseline, in the contralateral hemisphere. Finally, a general increase was observed in the power density of the low frequencies (theta and alpha) over all the scalp sites for both stimulation polarities, and a polarity-unspecific effect on behavioral performance, as evaluated by reaction times in a simple motor task.

\section{tDCS-induced changes in cortical excitability}

The tDCS is a weak current that can modify the trans-membrane neuronal potential, influencing the neuronal excitability threshold and modulating the firing rate of neurons in response to an input, as observed in animal studies (Bindman et al., 1964a; Purpura and McMurtry, 1965).

Considering that the cortical response to the TMS depends on the neuronal activation state (Amassian et al., 1989; Esser et al., 2006; Huber et al., 2008; Romei et al., 2008; Silvanto et al., 2008), the cortical response strength evoked by the TMS represents a direct measure of the neuronal changes induced by the tDCS on the motor cortex.

In our study, we clearly demonstrated an increase of the TEPs after the anodal stimulation and their reduction after the cathodal stimulation. On this basis, we hypothesize that the anodal tDCS decreases the synaptic activation threshold, increasing the probability that the TMS will polarize the cell membranes and, consequently, recruit a larger neuronal population. In addition, it determines the increase in the opening of the voltage-sensitive ion channels and the facilitation of action potential induction (Ilmoniemi and Kicic, 2010). Moreover, long-lasting neuroplasticity effects might be linked to enduring transformations of synaptic strength, which are likely related to the intracellular calcium concentration changes (Hattori et al., 1990; Islam et al., 1995). On the other hand, the observed decrease in the cortical reactivity after the cathodal tDCS represents the results of a reduced neuronal recruitment, due to an increased activation threshold (Komssi et al., 2004; Quartarone et al., 2006). This finding strengthens the hypothesis that the effects induced by the tDCS are partially localized intracortically (Lang et al., 2011), with a possible involvement of synaptic changes underlying the long lasting after-effects (Nitsche and Paulus, 2001; Nitsche et al., 2003b; Nitsche et al., 2005).

In this way, if the number of neurons recruited by a single TMS-pulse is directly related to their excitability and the amount of activity displayed on the scalp in terms of the strength of the evoked field reflects the synchronous activation of a neural population (Lehmann and Skrandies, 1980), then the changes observed in the cortical response after the tDCS are a clear expression of the differential neuronal recruitment by a single TMS-pulse, dependent on the current-polarity synaptic strength modulation.

The anodal tDCS - increasing the presynaptic discharge rate concurrently with the postsynaptic depolarization - could strengthen the synaptic connections, inducing an increased cortical evoked response with a probable concurrent involvement of the NMDA receptors (Islam et al., 1995; Nitsche et al., 2003a). Conversely, the reduction in presynaptic activity induced by the cathodal tDCS (combined with the postsynaptic hyperpolarization) could weaken the synaptic connections (Bindman et al., 1964a; Bindman et al., 1964b; Cheeran et al., 2008).

The modulations of the cortical reactivity, with the increase and decrease of peaks contained in the cortical response to the TMS after the anodal and cathodal stimulations respectively, provide evidence of a polarity-dependent involvement of both the excitatory and inhibitory postsynaptic potentials. Our results are in line with previous studies that, using the TMS-EEG, described the cortical plasticity changes induced by different neuroplasticity paradigms (Esser et al., 2006; Huber et al., 2008; Van Der Werf et al., 2006).

Although it is likely that these paradigms modulate several different interneuronal pools with similar neurophysiological outcomes (Cheeran et al., 2010), they share the same transient alteration of the cortical excitability. We suggest that these neurophysiological outcomes could be considered cortical excitability markers. Specifically, the increase of the cortical reactivity observed after the anodal tDCS resembles that observed after the high-frequency rTMS (Esser et al., 2006) and paired the associative stimulation at $25 \mathrm{~ms}$ (PAS-25) (Huber et al., 2008). In a similar manner, the cortical decrease after the cathodal tDCS resembles that observed after low-frequency rTMS (Van Der Werf et al., 2006) and PAS-10 (Huber et al., 2008), even if at different time intervals after the TMS pulse. Although the LMFP analysis does not allow us to identify a single TEP component and understand the functional meaning of its modulation after the application of the tDCS, the strong similarities between the polaritydependent cortical responses observed in our study and the cortical changes induced by other protocols (i.e., Huber et al., 2008), at early, middle and late time intervals observed over M1, could allow us to candidate the cortical activity modulations in those time windows as cortical plasticity markers in humans and to infer the involvement, at least in part, of similar underlying neuronal mechanisms. Moreover, if the cortical plasticity refers to the ability of the nervous system to change the effectiveness of the neural circuit transmission and that effect involves, in addition to other mechanisms, changes in the threshold for the initiation of an action potential, then the modulations induced by the tDCS on the TEPs could represent a clear and direct measure of cortical plasticity phenomena (Nitsche et al., 2003a). In this regard, we speculate that the anodal tDCS-induced potentiation of the neuronal response could be likely attributable to LTP-like mechanisms. Conversely, the neuronal depression induced by the cathodal tDCS could represent the cortical marker of LTD-like modifications. The use of cortical reactivity to evaluate the tDCS-induced effects 
rather than the peripheral output could allow one to assess the polarity-dependent modulations in silent cortical areas.

By comparing the cortical activation before and after the stimulation in both hemispheres, separately, for both the anodal and the cathodal polarity we observed two specific patterns of changes in the cortical excitability. After the anodal stimulation, we observed an increase in the cortical response not only over the stimulated cortex (Stagg et al., 2009a; Stagg et al., 2011) but also over the contralateral homotopic areas. This result is consistent with a recent study (Zheng et al., 2011) that reports a positive correlation between the stimulated motor cortex and the contralateral one, supporting a possible coupling of neuroactivity between motor regions (although in our study, the stimulated hemisphere was more strongly activated than the contralateral region). We suppose that the mechanisms responsible for this widespread and bihemispheric anodal effect (Lang et al., 2005; Pena-Gomez et al., 2011; Polania et al., 2010b) could involve current flowing in subcortical regions such as the thalamus (Bindman et al., 1962), which is the principal subcortical mediator of the TMS-contralateral response (Komssi et al., 2002). A tDCS-induced effect on the cortico-subcortical networks is supported also by recent evidence of a functional coupling increase on the thalamo-cortical circuits following anodal stimulation over the motor cortex (Polania et al., 2012). Moreover, we speculate that the not site-limited cortical excitability increase could be determined by a decrease of the contralateral hemisphere inhibition, mediated, at least partially, by the anodal tDCS-induced reduction of GABA concentration (Stagg et al., 2009b). A different pattern of cortical response was observed after the cathodal stimulation. Although the tDCS-induced modulation of the cortical activation was evaluated during a resting state, our results confirm a decrease over the stimulated cortex (Lang et al., 2005) and a contralateral increase in the cortical activation (Stagg et al., 2009a). Our merely speculative hypotheses regard a contralateral cortical compensation for an inhibitory stimulation as an adaptive response to neuronal interference (Stagg et al., 2009a) or, more simply, as an impairment of the interhemispheric inhibition (Vines et al., 2006).

Finally, the time-course of LMFP effects over the motor cortex highlight that the tDCS modulates, in a polarity-specific manner, not only the initial component of cortical response, that is the correlate of the direct activation of the stimulated area, but also the later components, that is the markers of the activity triggered by axonally conducted signals (Ilmoniemi and Kicic, 2010). The hypothesized mechanism underlying that time-course could be represented by a differential balance between the intracortical (presumably GABAergic) inhibition or an enhancement of the intracortical (presumably glutamatergic) excitation, or by a mix of both.

\section{tDCS-induced changes in cortical activity}

We observed tDCS-induced increases in theta and alpha power immediately after the tDCS application, both with the anodal and cathodal stimulations. This result agrees partially with the only study that analyzed cortical oscillations in response to tDCS applied over M1 during the resting state (Ardolino et al., 2005). Because anodal and cathodal tDCS induce opposite changes in the cortical excitability, we would have expected opposite changes in the underlying cortical neuronal activity. However, a previous study already reported that the two tDCS polarities induce the same cortical activation when investigated by magnetoencephalography (Venkatakrishnan et al., 2011). The lack of difference in the cortical oscillatory EEG observed in the current study is consistent with this evidence. The signal detected by EEG and analyzed in the frequency domain reflects the activity of a large cortical region and may be insensitive to subtle differences in the activation of the underlying excitatory or inhibitory neural populations. Although several studies have already evaluated tDCS-induced effects on the cortical oscillatory activity (Miniussi et al., 2012) we concluded, by directly comparing the different cortical measures acquired in this study, that the TEPs are more sensitive than the EEG power density to the possible tDCS modulatory effects induced on the cortical activity. The EEG oscillatory activity, however, could have been affected also by the peculiarities of the experimental design. The EEG activity during the resting state was not acquired immediately after the end of the tDCS application, but it was preceded by a TEP-MEP block, which lasted about 5 min. Thus, we cannot rule out the possibility that TEP-MEP measures affected the consecutive EEG activity. Furthermore, we should mention that the lack of specificity for the tDCS polarity could also reveal a general effect not related to stimulation, but simply to physiological modulations given the absence of a sham as a further control condition.

Polarity-specific effects were found at $30 \mathrm{~min}$ after the stimulation. A persistence of the alpha power density increase was observed after the anodal, but not after the cathodal stimulation. This result could suggest a different duration of the tDCS polarity-dependent long-term effects and thus a specific tDCS modulation on the oscillatory brain activity. In addition, a delta power density decrease was observed over the right hemisphere after the cathodal stimulation only. Nevertheless this effect, even if potentially interesting, should be taken with caution as it was not present at the short-term interval.

\section{tDCS-induced changes in behavior}

Evidence reported in the literature shows that tDCS applied to M1 can differentially affect the RTs: anodal tDCS applied at the vertex improves response times, if compared to cathodal tDCS (Elbert et al., 1981). Given that in the present study the tDCS was applied to the left M1 we would have expected polarity-specific effects mainly when participants performed the behavioral task using the right contralateral hand. Although a direct polarity effect on behavior cannot be concluded, participants showed faster RTs during the anodal stimulation compared to cathodal the tDCS when using the stimulated hand. Moreover, RTs negatively correlated with the MEP amplitudes in a way that was hand-specific, suggesting that a decrease in RTs was associated with an increase in the MEP amplitudes.

\section{Conclusion}

The present study demonstrates that combined TMS-EEG is a viable way to investigate the tDCS effects on the cortical reactivity directly, without the need of an activating task or a peripheral marker. Moreover, considering that the tDCS-induced MEP changes had a similar time course compared with the cortical reactivity changes, we hypothesized that differential changes in the cortical reactivity after the anodal and cathodal tDCS could provide a surrogate marker for neuromodulation induced by the tDCS over the stimulated area, and that they could represent an additional measure to disentangle the underlying mechanisms of the effects observed after the tDCS.

Although, the present study demonstrates also polarity-unspecific effects in the resting state oscillatory activity and in the behavioral performance, it provides for the first time that tDCS causes polaritydependent changes both in the cortical and corticospinal responses. Given the results, it is likely that the tDCS does not have a direct excitatory or inhibitory effect but mostly a modulation role, presumably expressed as to changes in the excitability of cortical circuits.

\section{Acknowledgments}

We thank Dr. Marta Bortoletto for helpful discussions. This research was supported by a Project grant from the "Ministero della Salute Ministry of Health" and from the Associazione Fatebenefratelli per la Ricerca (AFaR).

\section{Conflict of interest}

None. 


\section{References}

Amassian, V.E., Cracco, R.Q., Maccabee, P.J., 1989. Focal stimulation of human cerebra cortex with the magnetic coil: a comparison with electrical stimulation. Electroencephalogr. Clin. Neurophysiol. 74, 401-416.

Ardolino, G., Bossi, B., Barbieri, S., Priori, A., 2005. Non-synaptic mechanisms underlie the after-effects of cathodal transcutaneous direct current stimulation of the human brain. J. Physiol. 568, 653-663.

Arul-Anandam, A.P., Loo, C., 2009. Transcranial direct current stimulation: a new too for the treatment of depression? J. Affect. Disord. 117, 137-145.

Baudewig, J., Nitsche, M.A., Paulus, W., Frahm, J., 2001. Regional modulation of BOLD MRI responses to human sensorimotor activation by transcranial direct current stimulation. Magn. Reson. Med. 45, 196-201.

Bindman, L.J., Lippold, O.C., Redfearn, J.W., 1962. Long-lasting changes in the level of the electrical activity of the cerebral cortex produced by polarizing currents. $\mathrm{Na}-$ ture 196, 584-585.

Bindman, L.J., Lippold, O.C., Redfearn, J.W., 1964a. The action of brief polarizing currents on the cerebral cortex of the rat (1) during current flow and (2) in the production of long-lasting after-effects. J. Physiol. 172, 369-382.

Bindman, L.J., Lippold, O.C., Redfearn, J.W., 1964b. Relation between the size and form of potentials evoked by sensory stimulation and the background electrical activity in the cerebral cortex of the rat. J. Physiol. 171, 1-25.

Boggio, P.S., Nunes, A., Rigonatti, S.P., Nitsche, M.A., Pascual-Leone, A., Fregni, F., 2007. Repeated sessions of noninvasive brain DC stimulation is associated with moto function improvement in stroke patients. Restor. Neurol. Neurosci. 25, 123-129.

Bonato, C., Miniussi, C., Rossini, P.M., 2006. Transcranial magnetic stimulation and corticalevoked potentials: A TMS/EEG co-registration study. Clin. Neurophysiol 117 (8), 1699-1707.

Brunoni, A.R., Nitsche, M.A., Bolognini, N., Bikson, M., Wagner, T., Merabet, L., Edwards D.J., Valero-Cabre, A., Rotenberg, A., Pascual-Leone, A., Ferrucci, R., Priori, A., Boggio, P.S., Fregni, F., 2011. Clinical research with transcranial direct current stimulation (tDCS): challenges and future directions. Brain Stimul. 5 (3), 175-195.

Carducci, F., Brusco, R., 2012. Accuracy of an individualized MR-based head model for navigated brain stimulation. Psychiatry Res. 203, 105-108.

Casarotto, S., Canali, P., Rosanova, M., Pigorini, A., Fecchio, M., Mariotti, M., Lucca, A. Colombo, C., Benedetti, F., Massimini, M., 2013. Assessing the effects of electroconvulsive therapy on cortical excitability by means of transcranial magnetic stimulation and electroencephalography. Brain Topogr. 26 (2), 326-337.

Cheeran, B., Talelli, P., Mori, F., Koch, G., Suppa, A., Edwards, M., Houlden, H., Bhatia, K. Greenwood, R., Rothwell, J.C., 2008. A common polymorphism in the brain-derived neurotrophic factor gene (BDNF) modulates human cortical plasticity and the response to rTMS. J. Physiol. 586, 5717-5725.

Cheeran, B., Koch, G., Stagg, C.J., Baig, F., Teo, J., 2010. Transcranial magnetic stimulation: from neurophysiology to pharmacology, molecular biology and genomics. Neuroscientist 16, 210-221.

Cincotta, M., Giovannelli, F., Borgheresi, A., Balestrieri, F., Toscani, L., Zaccara, G., Carducci, F., Viggiano, M.P., Rossi, S., 2010. Optically tracked neuronavigation increases the stability of hand-held focal coil positioning: evidence from "transcranial" magnetic stimulation-induced electrical field measurements. Brain Stimul. 3, $119-123$

Creutzfeldt, O.D., Fromm, G.H., Kapp, H., 1962. Influence of transcortical d-c currents on cortical neuronal activity. Exp. Neurol. 5, 436-452.

Elbert, T., Lutzenberger, W., Rockstroh, B., Birbaumer, N., 1981. The influence of lowlevel transcortical DC-currents on response speed in humans. Int. J. Neurosci. 14 $(1-2), 101-114$

Esser, S.K., Huber, R., Massimini, M., Peterson, M.J., Ferrarelli, F., Tononi, G., 2006. A direct demonstration of cortical LTP in humans: a combined TMS/EEG study. Brain Res. Bull. 69, 86-94

Fertonani, A., Rosini, S., Cotelli, M., Rossini, P.M., Miniussi, C., 2010. Naming facilitation induced by transcranial direct current stimulation. Behav. Brain Res. 208 (2), $311-318$

Fricke, K., Seeber, A.A., Thirugnanasambandam, N., Paulus, W., Nitsche, M.A., Rothwell, J.C., 2011. Time course of the induction of homeostatic plasticity generated by repeated transcranial direct current stimulation of the human motor cortex. J. Neurophysiol. 105, 1141-1149.

Hattori, Y., Moriwaki, A., Hori, Y., 1990. Biphasic effects of polarizing current on adenosine-sensitive generation of cyclic AMP in rat cerebral cortex. Neurosci. Lett. 116, 320-324.

Huber, R., Maatta, S., Esser, S.K., Sarasso, S., Ferrarelli, F., Watson, A., Ferreri, F., Peterson, M.J., Tononi, G., 2008. Measures of cortical plasticity after transcranial paired associative stimulation predict changes in electroencephalogram slow-wave activity during subsequent sleep. J. Neurosci. 28, 7911-7918.

Ilmoniemi, R.J., Kicic, D., 2010. Methodology for combined TMS and EEG. Brain Topogr. $22,233-248$.

Ilmoniemi, R.J., Virtanen, J., Ruohonen, J., Karhu, J., Aronen, H.J., Naatanen, R., Katila, T., 1997. Neuronal responses to magnetic stimulation reveal cortical reactivity and connectivity. Neuroreport 8, 3537-3540.

Islam, N., Aftabuddin, M., Moriwaki, A, Hattori, Y, Hori, Y, 1995. Increase in the calcium level following anodal polarization in the rat brain. Brain Res. 684, 206-208.

Jang, S.H., Ahn, S.H., Byun, W.M., Kim, C.S., Lee, M.Y., Kwon, Y.H., 2009. The effect of transcranial direct current stimulation on the cortical activation by motor task in the human brain: an fMRI study. Neurosci. Lett. 460, 117-120.

Komssi, S., Kahkonen, S., 2006. The novelty value of the combined use of electroencephalography and transcranial magnetic stimulation for neuroscience research. Brain Res. Rev. 52, 183-192.
Komssi, S., Aronen, H.J., Huttunen, J., Kesaniemi, M., Soinne, L., Nikouline, V.V., Ollikainen, M., Roine, R.O., Karhu, J., Savolainen, S., Ilmoniemi, R.J., 2002. Ipsiand contralateral EEG reactions to transcranial magnetic stimulation. Clin. Neurophysiol. 113, 175-184.

Komssi, S., Kahkonen, S., Ilmoniemi, R.J., 2004. The effect of stimulus intensity on brain responses evoked by transcranial magnetic stimulation. Hum. Brain Mapp. 21, 154-164.

Kwon, Y.H., Ko, M.H., Ahn, S.H., Kim, Y.H., Song, J.C., Lee, C.H., Chang, M.C., Jang, S.H., 2008. Primary motor cortex activation by transcranial direct current stimulation in the human brain. Neurosci. Lett. 435, 56-59.

Lang, N., Siebner, H.R., Ward, N.S., Lee, L., Nitsche, M.A., Paulus, W., Rothwell, J.C., Lemon, R.N., Frackowiak, R.S., 2005. How does transcranial DC stimulation of the primary motor cortex alter regional neuronal activity in the human brain? Eur. J. Neurosci. 22, 495-504.

Lang N., Nitsche, M.A., Dileone, M., Mazzone, P. De Andres-Ares, J., Diaz-Jara, L., Paulus, W., Di Lazzaro, V., Oliviero, A., 2011. Transcranial direct current stimulation effects on I-wave activity in humans. J. Neurophysiol. 105, 2802-2810.

Lehmann, D., Skrandies, W., 1980. Reference-free identification of components of checkerboard-evoked multichannel potential fields. Electroencephalogr. Clin. Neurophysiol. 48, 609-621.

Liebetanz, D., Nitsche, M.A., Tergau, F., Paulus, W., 2002. Pharmacological approach to the mechanisms of transcranial DC-stimulation-induced after-effects of human motor cortex excitability. Brain 125, 2238-2247.

Mäki, H., Ilmoniemi, R.J., 2010. The relationship between peripheral and early cortical activation induced by transcranial magnetic stimulation. Neurosci. Lett. 478, 24-28 (30).

Miniussi, C., Thut, G., 2009. Combining TMS and EEG offers new prospects in cognitive neuroscience. Brain Topogr. 22, 249-256.

Miniussi, C., Cappa, S.F., Cohen, L.G., Floel, A., Fregni, F., Nitsche, M.A., Oliveri, M., Pascual-Leone, A., Paulus, W., Priori, A., Walsh, V., 2008. Efficacy of repetitive transcranial magnetic stimulation/transcranial direct current stimulation in cognitive neurorehabilitation. Brain Stimul. 1, 326-336.

Miniussi, C., Brignani, D., Pellicciari, M.C., 2012. Combining transcranial electrical stimulation with electroencephalography: a multimodal approach. Clin. EEG Neurosci. 43, 184-191.

Moliadze, V., Antal, A., Paulus, W., 2010. Electrode-distance dependent after-effects of transcranial direct and random noise stimulation with extracephalic reference electrodes. Clin. Neurophysiol. 121, 2165-2171.

Monte-Silva, K., Kuo, M.F., Liebetanz, D., Paulus, W., Nitsche, M.A., 2010. Shaping the optimal repetition interval for cathodal transcranial direct current stimulation (tDCS). J. Neurophysiol. 103, 1735-1740.

Monte-Silva, K., Kuo, M.F., Hessenthaler, S., Fresnoza, S., Liebetanz, D., Paulus, W., Nitsche, M.A., 2013. Induction of late LTP-like plasticity in the human motor cortex by repeated non-invasive brain stimulation. Brain Stimul. 6 (3), 424-432.

Nitsche, M.A., Paulus, W., 2000. Excitability changes induced in the human motor cortex by weak transcranial direct current stimulation. J. Physiol. 527 (Pt 3), 633-639.

Nitsche, M.A., Paulus, W., 2001. Sustained excitability elevations induced by transcranial DC motor cortex stimulation in humans. Neurology 57, 1899-1901.

Nitsche, M.A., Liebetanz, D., Antal, A., Lang, N., Tergau, F., Paulus, W., 2003a. Modulation of cortical excitability by weak direct current stimulation-technical, safety and functional aspects. Suppl. Clin. Neurophysiol. 56, 255-276.

Nitsche, M.A., Fricke, K., Henschke, U., Schlitterlau, A., Liebetanz, D., Lang, N., Henning, S., Tergau, F., Paulus, W., 2003b. Pharmacological modulation of cortical excitability shifts induced by transcranial direct current stimulation in humans. J. Physiol. 553, 293-301.

Nitsche, M.A., Liebetanz, D., Schlitterlau, A., Henschke, U., Fricke, K., Frommann, K., Lang, N., Henning, S., Paulus, W., Tergau, F., 2004. GABAergic modulation of DC stimulationinduced motor cortex excitability shifts in humans. Eur. J. Neurosci. 19, 2720-2726.

Nitsche, M.A., Seeber, A., Frommann, K., Klein, C.C., Rochford, C., Nitsche, M.S., Fricke, K., Liebetanz, D., Lang, N., Antal, A., Paulus, W., Tergau, F., 2005. Modulating parameters of excitability during and after transcranial direct current stimulation of the human motor cortex. J. Physiol. 568, 291-303.

Nitsche, M.A., Cohen, L.G., Wassermann, E.M., Priori, A., Lang, N., Antal, A., Paulus, W., Hummel, F., Boggio, P.S., Fregni, F., Pascual-Leone, A., 2008. Transcranial direct current stimulation: state of the art 2008. Brain Stimul. 1 (3), 206-223.

Oldfield, R.C., 1971. The assessment and analysis of handedness: the Edinburgh inventory. Neuropsychologia 9, 97-113.

Paquette, C., Sidel, M., Radinska, B.A., Soucy, J.P., Thiel, A., 2011. Bilateral transcranial direct current stimulation modulates activation-induced regional blood flow changes during voluntary movement. J. Cereb. Blood Flow Metab. 31, 2086-2095.

Pena-Gomez, C., Sala-Lonch, R., Junque, C., Clemente, I.C., Vidal, D., Bargallo, N., Falcon, C., Valls-Sole, J., Pascual-Leone, A., Bartres-Faz, D., 2011. Modulation of large-scale brain networks by transcranial direct current stimulation evidenced by restingstate functional MRI. Brain Stimul. 5 (3), 252-263.

Polania, R., Nitsche, M.A., Paulus, W., 2010a. Modulating functional connectivity patterns and topological functional organization of the human brain with transcranial direct current stimulation. Hum. Brain Mapp. 32, 1236-1249.

Polania, R., Paulus, W., Antal, A., Nitsche, M.A., 2010b. Introducing graph theory to track for neuroplastic alterations in the resting human brain: a transcranial direct current stimulation study. Neuroimage 54, 2287-2296.

Polania, R., Paulus, W., Nitsche, M.A., 2012. Modulating cortico-striatal and thalamocortical functional connectivity with transcranial direct current stimulation. Hum. Brain Mapp. 33, 2499-2508.

Priori, A., Berardelli, A., Rona, S., Accornero, N., Manfredi, M., 1998. Polarization of the human motor cortex through the scalp. Neuroreport 9, 2257-2260.

Purpura, D.P. McMurtry, J.G., 1965. Intracellular activities and evoked potential changes during polarization of motor cortex. J. Neurophysiol. 28, 166-185. 
Quartarone, A., Siebner, H.R., Rothwell, J.C., 2006. Task-specific hand dystonia: “can too much plasticity be bad for you?". Trends Neurosci. 29, 192-199.

Romei, V., Brodbeck, V., Michel, C., Amedi, A., Pascual-Leone, A., Thut, G., 2008. Spontaneous fluctuations in posterior alpha-band EEG activity reflect variability in excitability of human visual areas. Cereb. Cortex 18, 2010-2018.

Rossi, S., Hallett, M., Rossini, P.M., Pascual-Leone, A., Safety of TMS Consensus Group, 2009. Safety, ethical considerations, and application guidelines for the use of transcranial magnetic stimulation in clinical practice and research. Clin. Neurophysiol. 120, 2008-2039.

Rossini, P.M., Barker, A.T., Berardelli, A., Caramia, M.D., Caruso, G., Cracco, R.Q., Dimitrijevic, M.R., Hallett, M., Katayama, Y., Lucking, C.H., et al., 1994. Non-invasive electrical and magnetic stimulation of the brain, spinal cord and roots: basic principles and procedures for routine clinical application. Report of an IFCN committee. Electroencephalogr. Clin. Neurophysiol. 91, 79-92.

Sale, M.V., Ridding, M.C., Nordstrom, M.A., 2007. Factors influencing the magnitude and reproducibility of corticomotor excitability changes induced by paired associative stimulation. Exp. Brain Res. 181, 615-626.

Silvanto, J., Muggleton, N., Walsh, V., 2008. State-dependency in brain stimulation studies of perception and cognition. Trends Cogn. Sci. 12, 447-454.

Stagg, C.J., O'Shea, J., Kincses, Z.T., Woolrich, M., Matthews, P.M., Johansen-Berg, H., 2009a. Modulation of movement-associated cortical activation by transcranial direct current stimulation. Eur. J. Neurosci. 30, 1412-1423.

Stagg, C.J., Best, J.G., Stephenson, M.C., O'Shea, J., Wylezinska, M., Kincses, Z.T., Morris, P.G., Matthews, P.M., Johansen-Berg, H., 2009b. Polarity-sensitive modulation of cortical neurotransmitters by transcranial stimulation. J. Neurosci. 29, 5202-5206.

Stagg, C.J., Bachtiar, V., O'Shea, J., Allman, C., Bosnell, R.A., Kischka, U., Matthews, P.M., Johansen-Berg, H., 2011. Cortical activation changes underlying stimulationinduced behavioural gains in chronic stroke. Brain 135 (Pt 1), 276-284.
Van Der Werf, Y.D., Sadikot, A.F., Strafella, A.P., Paus, T., 2006. The neural response to transcranial magnetic stimulation of the human motor cortex. II. Thalamocortical contributions. Exp. Brain Res. 175, 246-255.

Van Der Werf, Y.D., Paus, T., 2006. The neural response to transcranial magnetic stimulation of the human motor cortex. I. Intracortical and cortico-cortical contributions. Exp. Brain Res. 175 (2), 231-245.

Veniero, D., Bortoletto, M., Miniussi, C., 2009. TMS-EEG co-registration: on TMS induced artifact. Clin. Neurophysiol. 120, 1392-1399.

Venkatakrishnan, A., Contreras-Vidal, J.L., Sandrini, M., Cohen, L.G., 2011. Independent component analysis of resting brain activity reveals transient modulation of local cortical processing by transcranial direct current stimulation. Conf. Proc. IEEE Eng. Med. Biol. Soc. 2011, 8102-8105.

Vernet, M., Bashir, S., Yoo, W.K., Perez, J.M., Najib, U., Pascual-Leone, A., 2013. Insights on the neural basis of motor plasticity induced by theta burst stimulation from TMS-EEG. Eur. J. Neurosci. 37 (4), 598-606.

Vines, B.W., Nair, D.G., Schlaug, G., 2006. Contralateral and ipsilateral motor effects after transcranial direct current stimulation. Neuroreport 17, 671-674.

Wachter, D., Wrede, A., Schulz-Schaeffer, W., Taghizadeh-Waghefi, A., Nitsche, M.A., Kutschenko, A., Rohde, V., Liebetanz, D., 2011. Transcranial direct current stimulation induces polarity-specific changes of cortical blood perfusion in the rat. Exp. Neurol. 227, 322-327.

Wagner, T., Valero-Cabre, A., Pascual-Leone, A., 2007. Noninvasive human brain stimulation. Annu. Rev. Biomed. Eng. 9, 527-565.

Wassermann, E.M., Grafman, J., 2005. Recharging cognition with DC brain polarization. Trends Cogn. Sci. 9, 503-505.

Zheng, X., Alsop, D.C., Schlaug, G., 2011. Effects of transcranial direct current stimulation (tDCS) on human regional cerebral blood flow. Neuroimage 58, 26-33. 